\title{
Observation of MEL stacking faults in two-dimensional MFI zeolite nanosheets
}

\author{
Prashant Kumar, ${ }^{1}$ Han Zhang, ${ }^{1}$ Neel Rangnekar, ${ }^{1}$ Michael Tsapatsis, ${ }^{1}$ K. Andre Mkhoyan ${ }^{1}$
}

${ }^{1}$ Department of Chemical Engineering \& Materials Science, University of Minnesota, Minneapolis, MN 55455 .

Two-dimensional (2D) MFI-type zeolite nanosheet has gained widespread recognition due to its commercial viability in several applications such as separations of difficult to separate hydrocarbon isomers like xylenes, selective reactions like sugar isomerizations and, dehydrations for the production of renewable fuels and chemicals [1-2]. These nanosheets are porous frameworks through which molecules of only appropriate size can pass through. Presence of any kind of defects distorts the pore structure thereby affecting their performance as separation membranes and catalysts. While, presence of stacking faults have previously been reported in MFI-type zeolite bulk crystals [3], no such observations have yet been made in 2D MFI frameworks. This study reports the observation of a different type zeolite i.e. MEL-type inter-grown within the MFI framework of the nanosheets, which appears as stacking faults (Figure 1).

In this work, bright field transmission electron microscopy (BF-TEM) was performed using FEI Tecnai G2 F30 (S)TEM equipped with TWIN pole piece and a Schottky field emission electron gun operating at $300 \mathrm{kV}$. Image were acquired using a Gatan $4 \mathrm{k} \times 4 \mathrm{k}$ Ultrascan CCD under low-dose TEM operating conditions to minimize structural damage of MFI framework.

MFI-type zeolite is composed of interconnected silicon and oxygen atoms forming periodic bond chains called as pentasil chains. These pentasil chains are parallel to [001] direction and interconnect with each other along [100] direction by inversion symmetry to form MFI-type framework as shown in Figure 2. However, a 90 degree rotation of the pentasil chain about [001] direction results in formation of a MELtype zeolite framework in which the pentasil chains are related by mirror symmetry (Figure 2). Observation of these defects in a MFI nanosheet leads to an unambiguous conclusion that MEL-type zeolite is present within a MFI-type zeolite nanosheets. In a separate study, it has already been shown, through TEM study, that these nanosheets are 1.5 unit-cell thick $(3.2 \mathrm{~nm})$ along [010] direction [4]. Furthermore, additional comparison of [010] zone axis electron diffraction patterns from the faulted nanosheet and pristine MFI-type nanosheet confirms our earlier high resolution TEM observations. It is observed that the diffraction spots of MFI-type lattice are streaked perpendicular to the stacking fault direction (Figure 3). Periodicity of these stacking faults and its effects on nanosheet performance during chemical separation will be further discussed [5].

\section{References:}

[1] M. Choi et al, Nature 461, (2009), 246-249.

[2] K. Varoon et al, Science 334, (2011), 72-75.

[3] G. R. Millward et al, Faraday Trans 2 79, (1983), 1075-1082.

[4] P. Kumar et al, Nat. Commun. 6, (2015), 7128.

[5] This work was supported as part of the Catalysis Center for Energy Innovation, an Energy Frontier Research Center funded by the U.S. Department of Energy, Office of Science, Basic Energy Sciences under Award DE-SC0001004. 


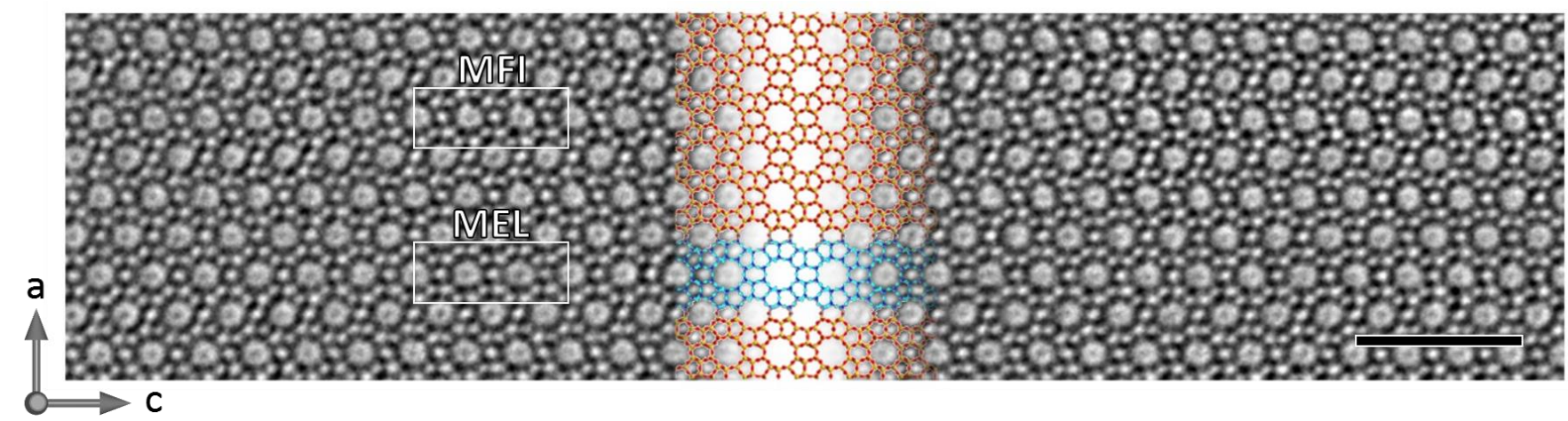

Figure 1. Weiner filtered high-resolution TEM image of a 1.5 unit-cell thick MFI-zeolite nanosheet along [010] zone axis, showing a stacking fault of MEL-type zeolite formed due to two pentasil chains related via mirror symmetry. Overlaid is the atomic structure highlighting pentasil chains related by inversion symmetry in red and yellow; pentasil chains related by mirror symmetry are shown in blue and violet. Scale bar is $4 \mathrm{~nm}$.
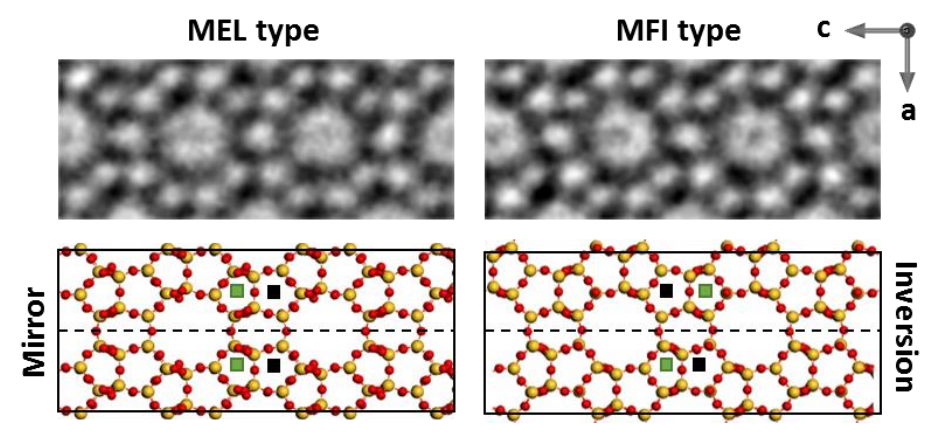

Figure 2. Sections of high resolution TEM image from Figure 1, showing two interconnected pentasil chains of MFI and MEL type zeolite. Dotted lines across the corresponding crystal structure represent the mirror plane in MEL and inversion centers in MFI.
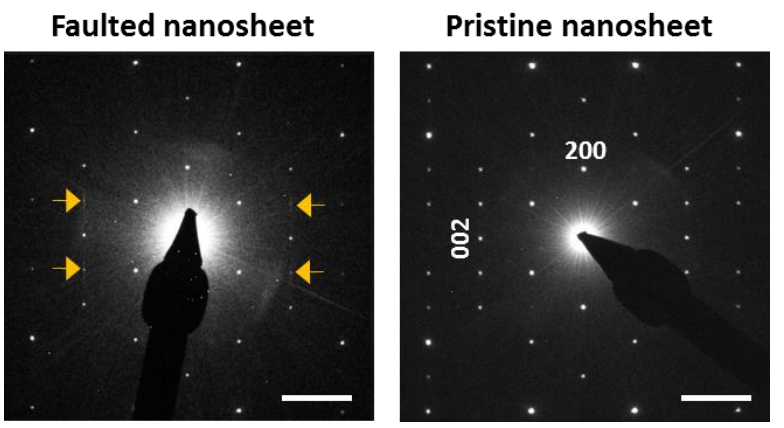

Figure 3. [010] zone axis diffraction pattern of the faulted nanosheet shown in Figure 1 has visible streaking across a periodic set of diffraction spots marked by yellow arrows. Pristine MFI nanosheet without any MEL stacking faults show no such spot streaking. Scale bars are $1 \mathrm{~nm}^{-1}$. 